\title{
Ein großes Werk zum Abschluss bringen
}

\begin{abstract}
After a short history of the Akademie-Ausgabe der Werke Immanuel Kants, it is shown that the edition is incomplete in some essential parts: The central topic of the first part of the article is the Opus postumum. Here we have a new critical transcription which differs not only in the composition but even in many details. The second project is focused on Nachschriften of Kant's lectures on Physische Geographie. The three volumes will show that Kant continually actualized the material for this complex area of problems, for which he presented the outlines of a new academic discipline (as he did too in his Anthropology). The other central topic is the new edition of the three Critiques. The second part of the article demonstrates principle steps of critical editing using the example of Kant's third Critique, the Critique of Judgment.
\end{abstract}

Keywords: Akademie Ausgabe, critical edition, Critique of Judgment, Kant, Opus postumum

Prof. Dr. Volker Gerhardt: Humboldt-Universität zu Berlin, Institut für Philosophie, Praktische Philosophie, Rechts- und Sozialphilosophie, Unter den Linden 6, Raum 3036a, 10099 Berlin; Volker.Gerhardt@Philosophie.hu-berlin.de

Dr. Jacqueline Karl: Berlin-Brandenburgische Akademie der Wissenschaften, Am Neuen Markt 8, 14467 Potsdam; karl@bbaw.de

Prof. Dr. Andrea Marlen Esser: Philips-Universität Marburg, Institut für Philosophie, WilhelmRöpke-Straße 6C, Turm C, Raum 01C03, 35032 Marburg; esser@staff.uni-marburg.de

\section{Zum Stand der Edition der Akademie-Ausgabe der Werke Immanuel Kants}

Eines der größten Philosophie-Institute Chinas befindet sich in den fünf oberen Stockwerken des Hauptgebäudes der Fudan-Universität in Shanghai. Auf der höchsten Ebene steht eine Platon-Büste. Eine Etage tiefer wird man von Immanuel Kant begrüßt. Darunter lächelt Konfuzius. Die Position auf der folgenden Etage ist nicht besetzt, doch auf der untersten Ebene des Instituts, im 22. Stock, in dem sich auch der Haupteingang befindet, steht die Büste von Karl Marx.

Spricht man mit den Philosophen in Shanghai, Beijing, Wuhan, Guangzhou oder Hangzhou, erfährt man, dass es für Marx zwar die großzügigste staatliche Förderung gibt, aber die Verlegenheit ist groß, weil man mit Marx-Studien in 
China schnell in Gegensatz zur gesellschaftlichen Realität gerät. Das Hauptinteresse der an der deutschen Philosophie interessierten Forschung gilt daher, neben Leibniz, Hegel und Nietzsche, dem Werk Immanuel Kants. Derzeit wird bereits die siebente Gesamtausgabe seiner Schriften vorbereitet. Mit der Neuübersetzung des Opus postumum wartet man bis zum Abschluss der Neuedition der BerlinBrandenburgischen Akademie der Wissenschaften (BBAW).

Das ist nur ein Schlaglicht auf die internationale Bedeutung Kants. Es ist müßig, seinen Rang gegenüber Platon, Aristoteles, Leibniz oder Hegel abzuwägen. Es genügt zu wissen, dass sein Werk überall auf der Welt studiert wird und dass es in so gut wie allen historischen und systematischen Fragen der philosophischen Forschung im Zentrum der Aufmerksamkeit steht. Damit ist klar, in welcher Verantwortung die BBAW durch ihr Privileg steht, die weltweit führende Edition der Werke ihres Mitglieds Immanuel Kant zu betreuen. Der Abschluss der 1894 in Angriff genommenen und durch die Ungunst der Geschichte mehrfach unterbrochenen, zeitweilig nach Göttingen ausgelagerten Akademie-Ausgabe der Werke Kants ist eine Aufgabe, an der sich auch die historische Legitimität der BBAW als Nachfolgerin der Preußischen Akademie der Wissenschaften erweist. Von Wilhelm Dilthey als „Musterausgabe“ für vergleichbare Editionen geplant, verwirklicht der Abschluss der Gesamtausgabe auch die ursprüngliche Intention der Preußischen Akademie: Das gesamte Werk eines großen Denkers als Grundlage für ein im Einzelnen wie im Ganzen umfassendes Verständnis zu vergegenwärtigen.

Um diesen Abschluss zu ermöglichen, hatte die Akademie 2001 ein zunächst auf zehn Jahre befristetes Akademievorhaben eingeworben, das überdies großzügig von der ZEIT-Stiftung Ebelin und Gerd Bucerius gefördert worden ist. Eine international besetzte Fachkommission und die in Potsdam angesiedelte Arbeitsstelle mit zwei wissenschaftlichen Mitarbeitern und einer wissenschaftlich-technischen Angestellten verantworten die Arbeit. Durch zusätzliche eingeworbene Mittel ist es zeitweilig möglich, weitere Fachkräfte zu verpflichten.

Mit der Übernahme der Editionsverantwortung durch die BBAW im Jahre 2001 haben sich die Arbeiten auf die Schließung der beiden größten Editionslücken konzentriert. Die bestanden bei den Vorlesungsnachschriften in der Physischen Geographie sowie in der notwendig gewordenen Neuedition der monumentalen Nachlass-Konvolute des greisen Kant im Opus postumum. Außerdem wurden (stets über zusätzliche Mittel - zuletzt auch von Jan Philipp Reemtsma finanziert) die drei Kritiken einer Revision unterzogen, die eine bereits jetzt als Meilenstein erkennbare Parallelpräsentation der beiden Auflagen der Kritik der reinen Vernunft zur Folge haben wird.

Die Arbeiten sind der Sache nach sehr gut vorangekommen, haben sich aber auf allen drei Feldern als wesentlich aufwändiger erwiesen. Von der Ausgabe 
der Vorlesungen über Physische Geographie, die im Gang der Edition durch eine weitere Nachschrift sowie durch weit verzweigte Quellenfunde auf drei Bände erweitert werden musste, liegt der erste Band seit 2009 vor; der auf zwei Teilbände aufgeteilte Band XXVI.2 kann wohl erst 2016 folgen. Die Verzögerungen haben sich dadurch ergeben, dass der für die Edition zuständige Werner Stark nicht nur eine verloren geglaubte Nachschrift der Physischen Geographie aus einem bislang gar nicht abgedeckten Zeitraum ausfindig machen konnte, das Manuskript Dönhoff, sondern auch Kants Handexemplar von Baumgartens Metaphysica (in der dritten Auflage von 1750) aufgefunden hat. An ihrer bereits in Arbeit befindlichen Neuausgabe, zusammen mit den zahlreichen Kommentaren Kants, ist Werner Stark auch beteiligt. Zudem wirkt er an der ebenfalls in Vorbereitung befindlichen separaten Neuedition von Herders Nachschriften der Vorlesungen Kants mit.

Die unerhört aufwändige Transkription des Opus postumum konnte erst nach zehnjähriger Arbeit abgeschlossen werden. Inzwischen arbeiten der Herausgeber, Eckart Förster, und die für die Transkription zuständige Arbeitsstellenleiterin, Jacqueline Karl, an der Erstellung einer lesbaren Fassung, die dann der Ergänzung durch die bereits weitgehend vorbereiteten Anmerkungen und Sachkommentare bedarf. Von der Schwierigkeit der hier bestehenden Aufgaben kann sich jeder selbst ein Bild machen, wenn er die im Netz verfügbaren Digitalisate der Faksimiles des Kantischen Manuskripts aufruft. Seit Ende letzten Jahres steht eine Online-Edition zur Verfügung, in der nicht nur die Transkription im Vergleich mit der Kantischen Handschrift zugänglich ist. Durch eine abschnittsweise wechselseitige Verlinkung von Transkription und Faksimile ist es möglich, sich ohne jede Einschränkung zwei Faksimileabschnitte auszuwählen und sich die Transkription beider Abschnitte gegenübergestellt anzeigen zu lassen. Die Online-Edition verbindet somit die lineare Textabfolge mit der Gleichzeitigkeit der Ansicht der Faksimileseite und ermöglicht eine durchgängige Orientierung über Ort und Zusammenhang der Textabschnitte auf der Manuskriptseite.

Die drei Kritiken werden nicht vor 2015 erscheinen können. Zwar sind die Vorbereitungen der Neuausgaben der Kritik der Urteilskraft (durch Andrea Marlen Esser) und der Kritik der praktischen Vernunft (durch Jens Timmermann) weit gediehen, doch das Ausscheiden der auch für die Koordination der Edition aller drei Kritiken zuständigen Herausgeberin der Kritik der reinen Vernunft hat einen Wechsel erzwungen, der einen zweijährigen Zeitverlust nach sich gezogen hat. Erst jetzt ist der Anschluss an die ebenfalls bereits weit fortgeschrittenen Vorarbeiten gefunden, so dass auch hier ein Ende der Arbeiten absehbar ist.

Allein die Hinweise auf die Gründe für die Verzögerung der ursprünglich geplanten Arbeiten lassen erkennen, dass die Akademie-Ausgabe der Werke Kants weder vervollständigt noch erneuert ist, wenn die ursprünglich vorgesehe- 
nen drei Aufgaben zum Abschluss gebracht worden sind. Schon kurz nach Aufnahme der Arbeiten in Potsdam hat sich gezeigt, dass noch einiges mehr zu tun ist, um die Ausgabe so abzuschließen, dass sie den Anforderungen der Kant-Forschung genügen kann und wieder das wird, was sie über einen langen Zeitraum war, aber leider zurzeit nicht mehr ist: nämlich die internationale Referenzausgabe für die wissenschaftliche Forschung.

Innerhalb der I. Abteilung Werke genügt es bei Weitem nicht, nur die drei Kritiken in revidierten Textfassungen vorzulegen. Es müssen vielmehr alle von Kant publizierten Schriften, die in der I. Abteilung der Edition versammelt sind, einer kritischen Durchsicht unterzogen werden. Gut hundert Jahre exegetischer Arbeit an Kants Schriften haben zahlreiche Emendationen der Textfassungen in der Akademie-Ausgabe ergeben; sie bedürfen längst der Aufnahme in eine Neuauflage der Gesamtausgabe, die endlich auch eine neue, alle Bände einbeziehende Studienausgabe möglich machen soll. Überdies stellt die Frakturschrift für viele Leser des angelsächsischen und insbesondere des asiatischen Bereichs ein ernstes Hindernis dar. Also muss die gesamte I. Abteilung überarbeitet und neugefasst werden. Schließlich bedürfen die Texte auch einer weltweit zugänglichen elektronischen Fassung, die den digitalen Umgang mit Kants Werk erleichtert.

Es war auch dieses vom Verlag Walter de Gruyter unterstützte Erfordernis, das den Anstoß zur Beantragung einer zweiten Arbeitsphase im Rahmen des Akademienprogramms gegeben hat. Sie ist Ende 2010 mit einer Laufzeit bis 2022 bewilligt worden. Inzwischen sind die Aufgaben für die Neuedition der nahezu 70 Texte in der I. Abteilung verteilt. Es haben sich 19 Editoren bereitgefunden, die Revision zu übernehmen. Nur für die naturwissenschaftlichen und geographischen Texte der Bände I und II sowie für die Metaphysischen Anfangsgründe der Naturwissenschaft hat sich noch kein Herausgeber gefunden. Der Arbeitsaufwand ist beträchtlich. Wenn es gelingt, zusätzliche Mittel für Hilfskräfte einzuwerben, die am Arbeitsort der Editoren verfügbar sind, müsste die Arbeit bis etwa $2018 \mathrm{zu}$ bewältigen sein.

Doch die Ausgabe ist damit noch nicht vollständig, weil weitere, erst in den letzten Jahrzehnten neu gefundene Kant-Texte fehlen. Dabei handelt es sich um für die Forschung essentielle Texte, die in einer Gesamtausgabe als solcher enthalten sein müssen. Geschieht dies nicht, gibt die Edition ihren Anspruch auf Vollständigkeit preis und kann nicht länger als leitende Edition gelten. Die deutsche Kant-Forschung hätte leichtfertig auf das Ziel einer Sicherung, Erschließung und Vergegenwärtigung eines bedeutenden kulturellen Erbes verzichtet.

$\mathrm{Zu}$ den bereits erwähnten Neufunden von Werner Stark gehört das Handexemplar Kants von Alexander Gottlieb Baumgartens Metaphysica (3. Aufl., 1750), nach der Kant über Jahre hinweg seine Metaphysik-Vorlesungen gehalten hat. Dieses mit leeren Seiten durchschossene Exemplar enthält auf fast sämtlichen 
Seiten handschriftliche Notate und Reflexionen Kants und ist auch deshalb so wichtig, weil die Einträge Rückschlüsse auf Kants Auseinandersetzung mit anderen philosophischen Autoren, insbesondere Baumgarten, in dieser frühen Zeit zulassen. Diese ein Buch füllenden Notizen sind bislang noch nicht publiziert. Sie bedürfen einer sorgfältigen Entzifferung, Zuordnung, Kommentierung und einer mit Baumgartens Vorlage verknüpften Edition im Rahmen der Akademie-Ausgabe.

Das Gleiche gilt für die Integration der seit 1955 neu gefundenen 32 Briefe sowie für die bislang noch unedierte umfangreiche amtliche Korrespondenz Kants, insbesondere die Texte aus und über Kants akademische Tätigkeit als Lehrer, Dekan und Rektor der Universität Königsberg. Aufgrund eines durchgeführten DFG-Forschungsprojekts an der Humboldt-Universität und eines ebenfalls durch Drittmittel finanzierten Forschungsvorhabens an der PhilippsUniversität Marburg können wir die noch nicht publizierten Materialien einschätzen. Sie gehören in eine Gesamtausgabe und werden ein bis zwei Bände füllen. Gleichzeitig gibt es eine Vielzahl verschiedener Unzulänglichkeiten in der Korrespondenz der vorliegenden Bände, die - zusätzlich zur Integration der fehlenden Texte - eine Revision der gesamten II. Abteilung Briefwechsel notwendig machen.

Zusätzlich haben die zuerst geplanten Neueditionen in die Struktur der bisherigen Ausgabe eingegriffen und machen erhebliche Revisionen nötig, um die Konsistenz der Gesamtausgabe zu wahren. Bei den Arbeiten an der Physischen Geographie hat sich gezeigt, dass die Nachschriften des genialen Kant-Schülers Johann Gottfried Herder nicht in die bisher übliche Ordnung der Nachschriften eingebracht werden können. Deshalb ist die frühe, für den Schüler entscheidende und für den jungen Lehrer kennzeichnende Nachschrift Herders nicht in den neuen Bänden der Physischen Geographie enthalten. Hier bedarf es einer separaten Ausgabe sämtlicher Vorlesungsnachschriften Herders, die für die Kantund für die Herder-Forschung von essenzieller Bedeutung ist.

Die Neuedition des Opus postumum wird ausschließlich den zum Nachlasswerk gehörenden Textbestand umfassen, nicht die im Manuskript ebenfalls enthaltenen Vorarbeiten zu anderen Werken, Briefentwürfen sowie Alltagsnotizen. Deshalb müssen diese Texte in einem neu zu edierenden Schlussband der III. Abteilung Handschriftlicher Nachlass aufgenommen werden, der zugleich die seit 1955 neu gefundenen sogenannten „Losen Blätter“ zu enthalten hat, sind sie doch als Notate zu und Entwürfe von späteren Werken wesentlich für das Verständnis der Genese des jeweiligen Werkes selbst. Andernfalls bliebe die Edition auch in diesem Punkt unvollständig.

Es fehlt schließlich noch ein sämtliche Bände umfassender Registerband, der die Vollständigkeit der Akademie-Ausgabe auch für den Nutzer erschließt. Die Tatsache, dass ein solches Register heute elektronisch zur Verfügung stehen 
muss, ist kein Argument gegen die Vorlage der Daten in einem abschließenden Band, der von denen erstellt werden sollte, die für den Abschluss der Ausgabe zuständig sind. Hier liegt die erkennbar letzte Aufgabe der Edition, die wir hoffentlich bis Ende 2022 zum Abschluss bringen können. ${ }^{1}$

Volker Gerhardt, Jacqueline Karl

\section{Die Neuedition der Critik der Urtheilskraft}

Kants Critik der Urtheilskraft wirft im Vergleich zu einigen anderen Werken Kants, über deren Textgestalt es jeweils eine lange Diskussion in der Kantforschung gibt (wie etwa im Fall der Metaphysik der Sitten, des Opus postumum oder der Vorlesungsnachschriften), weitaus weniger editorische Probleme auf. Es handelt sich bei der dritten Kritik ja ohne Frage um eine abgeschlossene Schrift, die noch zu Lebzeiten des Autors erschienen ist. In sie sind - mit Sicherheit noch bis in die zweite Auflage (1793) - Korrekturen und Verbesserungen eingegangen, die auf Veranlassung Kants oder zumindest mit seiner Autorisierung unternommen wurden.

Eine gründliche Revision und Neuedition auch dieses Textes ist dennoch dringend geboten. Seit den beiden Ausgaben der Kritik der Urteilskraft von 1908 und 1913, die von Wilhelm Windelband im Rahmen der „alten“ Akademieausgabe besorgt wurden, haben sich die wissenschaftlichen Ansprüche an eine historisch-kritische Ausgabe, aber auch die technischen Möglichkeiten, diesen Ansprüchen gerecht zu werden, gravierend gewandelt. Zum einen eröffnet die elektronische Verfügbarkeit weiter Teile des Kantischen Werks und zahlreicher zeitgenössischer Texte, auf die sie Bezug nehmen, viel umfangreichere und weitaus exaktere Recherche- und Überprüfungsverfahren, zum anderen haben sich die Rolle und das Selbstverständnis der Herausgebertätigkeit sehr grundlegend geändert. Während frühere Herausgeber zu ihren Aufgaben nicht nur die sprachliche Modernisierung, sondern auch die sachliche Glättung und sogar Vereinheitlichung der jeweiligen Textgrundlage zählten, begreifen heutige Herausgeberinnen und Herausgeber ihre Rolle weniger als „Vollstrecker“2 eines aufzuspürenden Autorenwillens. Sie verfolgen stattdessen das Ziel, mit einer Neue-

1 Zur Geschichte der Akademie-Ausgabe der Werke Kants vgl. z. B. Lehmann (1956), Heimsoeth (1957/58), Hinske (1990), Stark (1993), Brandt u. Stark (2000).

2 Plachta (2006), 91. 
dition eine Ausgabe bereitzustellen, die den Lesern zwar eine aufbereitete, aber hinsichtlich der editorischen Eingriffe und Veränderungen „transparente“ und in ihrer Genese nachvollziehbare Textfassung als Grundlage eigener Deutungsanstrengungen verfügbar macht. Daher sind sie in der Regel auch wesentlich zurückhaltender in ihren Eingriffen und entwickeln strenge formale Kriterien, etwa zur Definition eines Textfehlers, um das „emendatorische Vorgehen frei von ästhetischen und interpretatorischen Erwägungen $\mathrm{zu}$ halten““3. Die obersten Maximen solcher editorischer Tätigkeit bilden entsprechend die Wahrung der Texttreue und die Sparsamkeit in den verändernden und interpretationsbestimmenden herausgeberischen Eingriffen. Diese auf den ersten Blick scheinbar „positivistische“ Grundhaltung schließt freilich das Bewusstsein ein, dass der edierte Text trotz der genannten Maximen nach bestimmten Richtlinien hergestellt wurde und damit gleichwohl ein konstituierter Text ist. Das Ziel der Neuedition der Akademieausgabe und so auch der Critik der Urtheilskraft muss es daher sein, der Kantforschung eine Textgrundlage bereitzustellen, die dem skizzierten Stand der editionswissenschaftlichen Entwicklung entspricht und nach dessen Prinzipien erstellt worden ist. ${ }^{4}$ Den Aufwand, die Sorgfalt und die Komplexität der verschiedenen Arbeitsprozesse, die dazu notwendig sind, werde ich an einigen ausgewählten Beispielen aus der Neuedition der Critik der Urtheilskraft demonstrieren. Die Critik der Urteilskraft ist dazu deshalb gut geeignet, weil hier die einzelnen Schritte des Editionsprozess nicht in dem Sinne spektakulär sind, dass sogar der Text in einem buchtechnischen Sinne erst generiert werden muss. Dadurch treten die weniger spektakulären, aber nichtsdestoweniger wichtigen und folgenreichen Prozesse der Edition deutlicher hervor. Freilich zeigt schon der oberflächliche Vergleich der bisherigen Ausgaben der Critik der Urteilskraft, dass sie alle unter jeweils bestimmten, aber sich dabei durchaus unterscheidenden Editionsprinzipien verfertigt wurden. Und die Tatsache, dass diese Ausgaben sich jeweils entscheiden mussten, welche der vorliegenden autorisierten Textträger, d. h. welche der drei Originalausgaben, sie ihrer Edition zu Grunde legen, macht deutlich, dass es statt eines ursprünglich von Kant verfassten, aber als Autograph nicht mehr verfügbaren Textes eben nur diese drei nicht deckungsgleichen Versionen gibt. Aus ihnen eine gediegene Grundlage für die Kantforschung zu erarbeiten, stellt sich daher auch noch als Aufgabe für bereits mehrfach edierte Texte wie den der Critik der Urtheilskraft.

3 Scheibe (1982), 25.

4 Zum Beleg, dass dieses Ziel mit der vorliegenden Akademieausgabe nicht schon erreicht ist, vgl. Brandt (2000), der die Akademie-Ausgabe als einen „Palast [bezeichnet], der dringend saniert werden muss“ (VI). 


\section{Die Textkonstitution}

Zur Herstellung des Textes erarbeitete ein ganzes Team - an den Arbeiten waren Frau Anna Bouffier (RWTH Aachen), Frau Maja Schepelmann (ehemals RWTH Aachen, heute Universität Paderborn), Frau Dr. Tanja Gloyna (ehem. Herausgeberin CdrV), Herr Michael Hog und Herr Jörg Wissing (als Korrektoren) beteiligt aus der kritischen Sichtung und eingehenden Prüfung der drei autorisierten Originalausgaben (A-Auflage von 1790, B-Auflage von 1793 und C-Auflage von 1799) eine elektronische Textgrundlage in originaler Orthographie und Interpunktion. Das Ausgangsmaterial bildeten die elektronisch verfügbaren Daten des fünften Bandes der Akademie Ausgabe, die mit dem sogenannten Bonner Kant-Korpus zur Verfügung standen (http://korpora.zim.uni-duisburg-essen.de/kant/). Der gesamte Text der Critik der Urtheilskraft wurde zunächst vollständig in die Fassung der zweiten Originalausgabe, der B-Auflage (nach den Exemplaren des Marburger Archivs bzw. der Universitätsbibliothek Marburg sowie der Berliner Staatsbibliothek) zurückgesetzt. Die zweite Auflage wurde zur Textgrundlage bestimmt, weil sich für sie, nicht mehr aber mit Sicherheit für die dritte Auflage (C-Auflage von 1799), noch eine Einflussnahme Kants nachweisen lässt. Dieser Prozess der Zurücksetzung lässt sich zwar hier in einem Absatz skizzieren, er vollzieht sich aber tatsächlich in einem monatelangen, durchaus mühevollen, kooperativen Arbeitsprozesses, der größte Konzentration und Aufmerksamkeit erfordert - ein Prozess, der aber immerhin schon viele offensichtliche Fehler der bisherigen Textgrundlage der Akademie Ausgabe ans Licht brachte.

In einem zweiten Schritt wurden die Varianten der Ausgaben A (1790) (Universitätsbibliothek FU Berlin) und C (1799) (ebenfalls Universitätsbibliothek FU Berlin) von meiner Mitarbeiterin Frau Schepelmann in einer Variantentabelle und in einer synoptischen Übersicht ${ }^{5}$ vollständig erfasst; auf dieser Grundlage konnten schon einmal erste, allerdings noch vorläufige Regeln zur Verzeichnung der Varianten in der Neuedition gewonnen werden, die dann im editorischen Bericht aufgelistet bzw. unter den „Beobachtungen zur Sprache“ mitgeteilt werden. Die Ausdifferenzierung und Überprüfung dieser Regeln war und ist ein Prozess, der während der ganzen Bearbeitung des Textes fortdauert. Zum einen, weil die Regeln mit den Herausgebern der anderen beiden Kritiken und ihren Vorschlägen abgestimmt werden mussten, zum anderen, weil sich bei der Revision des Textes die Frage nach weiteren und umfassenderen Reduktionsmög-

5 Eine synoptische Darstellung fasst mehrere Bearbeitungsstufen (hier also A, B und C-Auflage) in einer parallelen Anordnung zusammen, so dass sie auf einen Blick zu erfassen sind (vgl. auch Plachta 2006, 106). 
lichkeiten immer wieder aufs Neue stellte, um den philologischen Apparat nicht zu überlasten und trotzdem zu einer aussagekräftigen Variantenverzeichnung zu gelangen. Varianten, die für das Verständnis und die Interpretation folgenreich sein können, sollten auch nicht in einer Flut wenig sinnverändernder Einträge untergehen. Reduktionen ließen sich zwar leicht für die Fälle vornehmen, in denen die Veränderungen zwischen den Ausgaben Regelmäßigkeiten aufwiesen oder lediglich sprachliche Modernisierungen waren. Doch dieses eher mechanischen Vorgehen musste durch weitere Überlegungen ergänzt werden, wie etwa durch die, dass Varianten an bestimmten Stellen, auch wenn es sich um Fälle regelmäßiger Abweichung oder sogar Fehler handelte, doch verzeichnet werden sollten, weil und wenn zu vermuten war, dass ein Leser der anderen beiden Auflagen im Textverständnis ohne den Hinweis auf die Varianz missgeleitet werden könnte. An vielen Stellen (zum Beispiel an der Stelle AA 5: 366, 21-22: B-Auflage: „Grund desselben in“, A-Auflage: „Grund derselben in“), macht die Korrektur in B einen grammatischen Bezug klar, der in A sogar offensichtlich fehlerhaft, aber auch noch in B missverständlich sein könnte. Mit der Verzeichnung des fehlerhaften Bezugs wird auch korrekte Bezug in B dadurch noch einmal klarer, weil er für den Leser als eine explizit durchgeführte Korrektur sichtbar wird.

Das Ziel der Variantenverzeichnung ist es, dass sich die Leser über den Blick in den Apparat auch die anderen Auflagen erschließen und einen Eindruck von ihrem Zustand und Charakter gewinnen können (etwa über den „elenden“ der A-Auflage ${ }^{6}$ und den besonderen Stil der Korrekturen der C-Auflage). Besondere Herausforderungen stellen dabei freilich die Stellen dar, in denen die Textvarianz semantisch widersprechende Wendungen präsentiert und dadurch die Editorin zu einer interpretatorischen Stellungnahme zwingt. Das ist etwa bei der berühmten so genannten „Euler-Stelle“ (AA 5: 224) der Fall: Hier ist mit der editorischen Entscheidung unweigerlich auch eine sachliche Entscheidung der in der Kantforschung diskutierten Frage verbunden, ob Kant nun an der Undulationstheorie Eulers „gar sehr“ (A und B) oder „gar nicht“ (C) zweifelt. Die Emendation des Textes, die ich an dieser Stelle - wie Windelband auch - schon mit der Variante der dritten Auflage (C) vorgenommen habe, wird daher in einer Sachanmerkung begründet und mit den Stellen in Kants Schriften gestützt, die für das Votum maßgeblich waren. Wichtig ist dabei freilich, dass die Belege für die Richtigkeit der Korrektur an A und B durch C aus dem Text der Critik der Urtheilskraft stammen und dann freilich auch nachgewiesen wird, dass die Position auch in anderen Schriften Kants, wie etwa in De igne, in den Metaphysischen Anfangsgründen der Naturwissenschaft und in der Anthropologie in pragmatischer Hinsicht von ihm

6 Vgl. Erdmann (1880), XI. 
vertreten wird. Aus der Sichtung aller Stellen, die das Thema behandeln, wird deutlich, dass Kant nicht daran zweifelt, dass die „Form in der Verbindung verschiedener Vorstellungen wahrgenommen werden kann“ (obwohl man darüber nicht ganz sicher sein kann, vgl. 5: 324,28). Sowohl in 5: 324,31 als auch in 5: 325,14 ist von „Form“ (im „Spiel der Erkenntnisvermögen“) die Rede, die in der ästhetischen Beurteilung gefallen soll, also durch Reflexion, nicht durch Empfindung ins Urteil Eingang findet - das kann allerdings nur der Fall sein, wenn eine entscheidende Bedingung erfüllt ist: dass es eine Regelmäßigkeit im Spiel der Eindrücke gibt (vgl. 5: 224,26).

Die Konjekturen der einschlägigen anderen Ausgaben (also die eigenständigen Verbesserungen des Textes, die von früheren Herausgebern getätigt worden sind) wurden, sofern es sich tatsächlich um Konjekturen und nicht nur um stilistische oder modernisierende Eingriffe handelte, in einer Tabelle zusammengefasst, die insgesamt 674 Positionen umfasst und die Ausgaben von Rosenkranz (1838), Hartenstein 1(1839), Hartenstein 2 (1867), Kirchmann (1869), Kehrbach (1878), Erdmann (1880), Vorländer 3 (1902), Vorländer 4 (1913), Windelband AA (1908/1913), Cassirer/Buek (1914,1922), Schmidt (1930), Weischedel (1957), Lehmann (1963), Frank/Zanetti (1996) und Klemme (2001, 2003, 2006) einschließt. Frau Schepelmann hat dazu in einem ersten Schritt die textkritischen Apparate der anderen Ausgaben ausgewertet und die jeweiligen Ergebnisse dieses ebenfalls sehr mühevollen und ausgesprochen zeitaufwendigen Prozesses dann in einem zweiten Durchgang „im Krebsgang“ an den Apparaten und bei Abweichungen an den jeweiligen Editionen noch einmal überprüft. Verzeichnet wird nur der jeweils erste Vorschlag; in die Neuedition übernommen werden Konjekturen anderer Herausgeber und Konjekturen, die mit einer Variante durchgeführt werden, nur dann, wenn der Text andernfalls grammatikalisch falsch, stilistisch problematisch bzw. von den üblichen sprachlichen Gepflogenheiten des Autors abweichend, missverständlich oder unverständlich wäre, oder wenn er sachlich in offensichtlich grober Spannung zu einer Vielzahl anderen Stellen stehen würde. ${ }^{7}$ In den Sachanmerkungen werden auch Konjekturvorschläge der Forschungsliteratur berücksichtigt (wie etwa im Falle der oben genannten Stelle in AA 5: 224, „gar nicht“; „gar sehr“ oder im Fall der von Kiesewetter vorgenom-

7 Zur Bestimmung dessen, was als Fehler etc. jeweils gelten kann, wurden auf der Grundlage heute üblicher editionswissenschaftlicher Verfahren Kriterien entwickelt und jeweils auf dem Wege umfassender Untersuchungen Nachweise vorgelegt betreffend den Sprachgebrauch des 18. Jhd., den kantischen Sprachgebrauch, den Bezug auf Parallelstellen oder analoge Argumentionen, die mit der Neuedition elektronisch zugänglich gemacht werden sollen. 
menen Korrektur AA 5: 309, 33-34 von „Nachahmung“ in „Nachmachung“; vgl. Brief von Kiesewetter an Kant vom 3.3.1790, AA 11: 138).

Die genaue Sichtung und Überprüfung der anderen Ausgaben hat interessante Einblicke in die frühere Editionspraxis eröffnet. Zum einen ist deutlich geworden, wie viele Fehler in den Apparaten über viele Ausgaben hindurch unkorrigiert von Edition zu Edition weitergegeben wurden, weil die Apparate einfach abgeschrieben worden sind. Zum anderen konnte Frau Schepelmann belegen, dass die Ausgabe Hartensteins entgegen der Angabe des Herausgebers gar nicht nach der zweiten, sondern tatsächlich überwiegend nach der dritten Auflage ediert hat. Dasselbe lässt sich bei kritischer Durchsicht der Ausgabe von Kirchmann bemerken: Von Kirchmann ediert nach der Vorlage Hartensteins, legt also ebenfalls die dritte Originalausgabe zu Grunde und nicht die zweite. Darauf hat bereits Kehrbach in dem Vorwort zu seiner Ausgabe von 1878 hingewiesen: „Uebrigens wird eine Vergleichung der in vorliegender Ausgabe verzeichneten Varianten von C mit dem Hartenstein'schen und Kirchmann'schen Text das Resultat ergeben, daß diesen beiden Texten nicht wie ausdrücklich angegeben B, sondern C zu Grunde gelegen haben muß“8 . In der Folge wird die Neuedition statt Hartenstein in jenen Fällen, in denen der Text der zweiten Auflage mit der dritten konjiziert wird, Erdmann als den nennen, der dies als erster unternommen hat. Erdmann war es auch, der schon früh darauf hingewiesen hat, dass man die Ziele, die Kant in seinen Werken verfolgt, nicht erfassen und verstehen kann, ohne die historischen Bedingungen einzubeziehen, aus denen sie hervorgegangen sind. ${ }^{9}$ Diese Einsicht betrifft sowohl den allgemeinen zeitgenössischen Diskussionshintergrund (die sogenannten „Folien“), der freilich im Rahmen der Edition nicht erschlossen werden kann, als auch die direkten und indirekten Bezugnahmen Kants auf die damals aktuellen Diskussionszusammenhänge in der Ästhetik des Schönen und Erhabenen, in den Debatten über den Status und die Bedeutung organisierter Wesen und in der Physikotheologie, die in den Sachlichen Anmerkungen entschlüsselt werden sollen.

\section{Sachliche Anmerkungen}

Für die sachlichen Anmerkungen haben Frau Dr. Ulrike Santozki (ehem. Marburg) und Frau Schepelmann Vorarbeiten geleistet und erste Recherchen unternommen. Herr apl. Prof. Dr. Andreas Eckl (Frankfurt) hat die Kommentierungen aller bisherigen nationalen und internationalen Ausgaben Ausgaben

8 Kehrbach (1878), VIII.

9 Vgl. dazu Waschkies (1987), 45, mit Bezug auf Campo (1959), XXIX-XXXIII. 
erfasst und daraufhin geprüft, ob sie entsprechend der Editionsrichtlinien relevant und sachlich korrekt sind sowie Kants implizite Bezugnahmen auf Hume, Newton und die naturwissenschaftliche Forschung seiner Zeit erschlossen. Die sachlichen Anmerkungen der Neuedition werden, der Tradition der Akademieausgabe weiter folgend, knapp, informativ, nicht aber enzyklopädisch sein und sollen nur auf diejenigen Materialien hinweisen, die zum Verständnis der jeweiligen Stelle beitragen. Die Minimalität der Kommentierung soll die wissenschaftliche Langlebigkeit garantieren, die von historisch kritischen Ausgaben intendiert ist. Damit die Ausgabe aber dennoch „,benutzerfreundlich“ ist, werden Stellen mit Anmerkungen versehen, die auf Grund der Knappheit der Argumentation oder der sprachlichen Darstellung nur schwer verständlich sind oder die allein durch Verweis auf die Quellen der Hintergrunddiskussion erschlossen werden können. Die sachlichen Anmerkungen umfassen also Wort und Sacherläuterungen zu Personen, direkten oder indirekten Zitaten und Quellennachweise, Anspielungen und Hinweise auf wichtige, texterschließende Parallelstellen. Insbesondere die Critik der Urteilskraft versorgt den Leser mit einer ganzen Fülle von indirekten Bezügen auf aktuelle Diskussionszusammenhänge, aber darüber hinaus auch mit Bezügen auf Beispiele, die Kant schon in seinen frühen Schriften zur Verdeutlichung seiner Überlegungen herangezogen hat. Die nun auch schon jahrelange Arbeit an den sachlichen Anmerkungen zur Critik der Urtheilskraft hat gezeigt, dass Kant darin viele Themen, an denen er schon seit frühen Jahren arbeitete, in einem systematischen Zusammenhang zu verbinden versucht. Sowohl in der Ästhetik als auch in der Teleologie finden sich Sätze und mitunter auch ganze Passagen, die bis in den Wortlaut und die Formulierung hinein anderen Passagen aus früheren, teils sogar „vorkritischen“ Schriften (wie etwa aus der Schrift Allgemeine Naturgeschichte und Theorie des Himmels von 1755 oder aus Der einzig mögliche Beweisgrund zu einer Demonstration des Daseins Gottes von 1763) gleichen. Durch die Platzierung solcher Passagen in dem neuen Zusammenhang, insbesondere von Passagen aus vorkritischen Schriften in den Zusammenhang „kritischer“ Argumentationen verändert sich ihre Bedeutung freilich gravierend. In manchen Fällen sind dagegen bestimmte Passagen in der Critik der Urtheilskraft argumentativ so knapp gehalten, dass sie in ihrer Funktion für den Gedankengang nicht verständlich werden, wenn man nicht jene, oft ebenfalls bis in den Wortlaut gleiche, aber dann in der Argumentation umfassendere Passagen hinzuzieht. Solche Querverweise innerhalb des Kantischen Werks sollen helfen, den Text zu erschließen; weil sie aber das Textverständnis unweigerlich prägen, werden sie nur unter der Bedingung angegeben, dass auch philologisch nachweisbare Bezüge bestehen und werden auch nur an solchen Stellen angegeben, an denen es sich a) um ein Selbstzitat Kants handelt oder b) dieses Selbstzitat auf Argu- 
mentationen früherer Schriften verweist, durch die es gelingt, die Bedeutung der Stelle für den Gedankengang in der Critik der Urteilskraft zu erschließen. Die „Weiterverwendung“ von Passagen, die sich bis in die Formulierung hinein gleichen, eröffnet allerdings auch wichtige Einblicke in die Arbeitsweise Kants und in die Entwicklung des Werkes selbst. Mit dem Nachweis der sprachlichen, gedanklichen und argumentativen Vernetzung der Critik der Urtheilskraft sogar mit frühen, thematisch verwandten Schriften arbeitet diese Edition der nicht immer produktiven Tendenz in der aktuellen Kantforschung entgegen, die Werke Kants und sogar noch einzelne Argumentationen in den Werken nur isoliert $\mathrm{zu}$ bearbeiten und sie aus der Verwobenheit und den engen, eben oft auch sachlich und argumentativ einander ergänzenden Bezügen mit anderen Schriften herauszulösen. Vor allem in der Critik der Urtheilskraft scheint Kant aus der Fülle der von ihm bereits erarbeiteten Diskussionshintergründe $\mathrm{zu}$ schöpfen und sie durch Bezüge zur aktuellen Diskussion (insbesondere in der Teleologie) zu ergänzen. Um diese Hintergründe zu erschließen, ist eine nach heutigen Maßstäben wenig ökonomische Arbeitsform erforderlich: die aufmerksame Lektüre von schier nicht enden wollenden Materialien, die aber eben nicht mechanisch „durchsucht,“ sondern unter höchster Aufmerksamkeit und bester Kenntnis des kantischen Textes durchgearbeitet werden müssen - eine Arbeit, die nicht delegiert werden kann.

Dennoch bleibt die Edition ein kooperatives Unternehmen und ist daher wie kaum ein Forschungsprojekt auf die Kontinuität der Zusammenarbeit angewiesen, aber auch auf kontinuierliche Beschäftigung, um die mühevoll erarbeiteten Zusammenhänge präsent zu halten und die Lese- und Sichtungsarbeit überhaupt leisten zu können. Dass dazu die unsichere Finanzierungssituation (in unserem Fall haben die „Patchworkfinanzierung“ und die damit verursachten Unterbrechungen es der Herausgeberin der Critik der reinen Vernunft unmöglich gemacht, ihre Arbeit abzuschließen) und die universitäre Alltagssituation mit ihrer hohen Arbeitsbelastung nicht nur nicht förderlich, sondern hinderlich und hemmend sind, dürfte ohne weitere Erläuterung nachvollziehbar sein. Für eine angemessene Wertschätzung und damit vielleicht auch für eine größere Unterstützung dieser „Schildkrötenprojekte“ wäre es sicher wichtig, auch schon im Rahmen der universitären Lehre Einblick in die Arbeit der Edition und in ihre konkreten Arbeitsvollzüge zu geben, um auf diese Weise dem wissenschaftlichen Nachwuchs zu vermitteln, dass der scheinbar nur „historische“ Gegenstand seinerseits das Produkt einer zu seiner Entstehungszeit lebhaften, systematischen Auseinandersetzung mit den zeitgenössischen Diskussionen ist und auch er, wie jeder Gegenstand der Philosophie, zum Gegenstand einer kritischen Reflexion gemacht werden muss. 


\section{Literatur}

Brandt, R. u. Stark, W. (Hg.) (2000), Zustand und Zukunft der Akademie-Ausgabe von Immanuel Kants gesammelten Schriften (Kant-Studien 91, Sonderheft).

Campo, M. (1959), La genesis del criticismo kantiano, Varese.

Erdmann, B. (1880), Einleitung zu Immanuel Kant's Kritik der Urtheilskraft, Leipzig.

Heimsoeth, H. (1957/58), Zur Akademieausgabe von Kants gesammelten Schriften. Abschluß und Aufgaben, in: Kant-Studien 49, 1957/58, 351-363.

Hinske, N. (1990), Die Kantausgabe der Preußischen Akademie der Wissenschaften und ihre Probleme, in: Il cannocchiale. Rivista di studi filosofici 3, Neapel, 229-254.

Kehrbach, K. (1878), Kritik der Urteilskraft, Vorrede, Leipzig.

Lehmann, G. (1956), Zur Geschichte der Kant-Ausgabe 1896-1955, in: Deutsche Akademie der Wissenschaften zu Berlin 1946-1956, Berlin, 422-434.

Plachta, B. (2006), Editionswissenschaft, Stuttgart.

Scheibe, S. (1982), Zum Editorischen Problem des Textes, in: Zeitschrift für Deutsche Philologie 101 (Sonderheft: Probleme neugermanistischer Edition).

Stark, W. (1993), Nachforschungen zu Briefen und Handschriften Immanuel Kants, Berlin.

Waschkies, H.-J. (1987), Physik und Physikotheologie des jungen Kant, Bochumer Studien zur Philosophie. 